\title{
SUPPLEMENTAL MATERIAL OF RANDOM-EFFECTS META-ANALYSIS OF PHASE I DOSE-FINDING STUDIES USING STOCHASTIC PROCESS PRIORS
}

1. Bibliography search and trials details: sorafenib example.

We have used "sorafenib" in the "Other terms" search box of clinicaltrials.gov on June 30, 2019. Then we downloaded the 833 references and selected:

- Interventions:

- Drug: sorafenib

- Drug: Sorafenib

- Drug: Sorafenib Tosylate

- Drug: Nexavar|Drug: Sorafenib

- Drug: Sorafenib (BAY43-9006) Film-coated tablet|Drug: Sorafenib (BAY43-9006) Oral suspension

- Drug: Sorafenib dose escalation

- Drug: Sorafenib (Nexavar, BAY43-9006)

- Drug: Nexavar

- Drug: Nexavar (Sorafenib, BAY43-9006)

- Drug: BAY 43-9006

- Drug: Sorafenib $100 \mathrm{mg}$ (50-mg tablet)|Drug: Sorafenib $200 \mathrm{mg}$ (50-mg tablet)|Drug: Sorafenib $400 \mathrm{mg}$ (50-mg tablet)|Drug: Sorafenib 400 mg (200-mg tablet)|Drug: Sorafenib 400 mg (Expansion)

- Drug: Nexavar (BAY 43-9006) (Sorafenib)|Drug: Toxicity, Pharmacokinetics|Drug: Pharmacodynamics|Drug: Radiographic Evaluation|Drug: QOL assessment, Neuropsychological

- Phases:

- Early Phase I

- Phase I

- Phase I/Phase II 
The following 36 NCT numbers resulted from the search: NCT02636426, NCT02599337, NCT01561833, NCT00997022, NCT01398501, NCT02538393, NCT00954278, NCT00610246, NCT00892658, NCT00884416, NCT00572078, NCT00562523, NCT00622479, NCT01337492, NCT00606125, NCT00562913, NCT00259129, NCT00452218, NCT00287495, NCT00941863, NCT00663741, NCT00727233, NCT00829231, NCT00892424, NCT00541840, NCT01271504, NCT00864032, NCT00999843, NCT00414388, NCT00767468, NCT00093613, NCT00405366, NCT00118170, NCT00131989, NCT00217646, NCT00436579.

Table 1 shows the conditions treated and the cycle definition in each study resulted by the search on PubMed. Unfortunately not all trials have published their NCT number (only Borthakur et al. 2011 (NCT00217646) and Chen et al. 2014 (NCT01398501)), therefore it is complicated to state how many of them are included in the results of the clinicaltrials.gov search. The papers that did not report the registration number were published online before 2010 when there was no obligation to do so. Since then, the ICMJE (International Committee of Journal medical editors) requires and recommends that all medical journal editors request registration of clinical trials in a public trials registry at or before the time of first patient enrollment as a condition of consideration for publication. 
TABle 1

Details on conditions and cycle definition for the 14 studies on sorafenib monotherapy.

The drug was administered twice a day in all studies.

\begin{tabular}{|c|c|c|}
\hline Study & Conditions & Cycle information \\
\hline Clark et al. (2005) & Advanced, Refractory Solid Tumors & $\begin{array}{l}\text { Repeated cycles of } 1 \text { week } \\
\text { on and } 1 \text { week off }\end{array}$ \\
\hline Awada et al. (2005) & Advanced, Refractory Solid Tumors & $\begin{array}{l}\text { Repeated cycles of } 21 \text { days } \\
\text { on } / 7 \text { days off }\end{array}$ \\
\hline Moore et al. (2005) & Advanced, Refractory Solid Tumors & $\begin{array}{l}\text { repeated cycles of } 35 \text { days } \\
\text { ( } 28 \text { days on } / 7 \text { days off })\end{array}$ \\
\hline $\begin{array}{l}\text { Strumberg et al. } \\
(2005)\end{array}$ & Advanced, Refractory Solid Tumors & Varying weekly schedule \\
\hline Minami et al. (2008) & Advanced, Refractory Solid Tumors & $\begin{array}{l}\text { A single dose followed by a } \\
\text { 7-day wash-out period, and } \\
\text { then administrated twice } \\
\text { daily (bid) }\end{array}$ \\
\hline Miller et al. (2009) & $\begin{array}{l}\text { Solid tumors, multiple myeloma, or non- } \\
\text { Hodgkins lymphoma; Patients with Hep- } \\
\text { atic or Renal Dysfunction }\end{array}$ & $\begin{array}{l}\text { a single dose of } 400 \mathrm{mg} \text {, } \\
\text { then it is administrated } \\
\text { twice daily (bid) from day } \\
8\end{array}$ \\
\hline Nabors et al. (2011) & $\begin{array}{l}\text { Recurrent or progressive malignant } \\
\text { glioma }\end{array}$ & 28-day cycles \\
\hline Chen et al. (2014) & $\begin{array}{l}\text { Allogeneic Hematopoietic Stem Cell } \\
\text { Transplantation for Fms-like Tyrosine } \\
\text { Kinase } 3 \text { Internal Tandem Duplication } \\
\text { Acute Myeloid Leukemia }\end{array}$ & 12 28-day cycles \\
\hline Jia et al. (2013) & $\begin{array}{l}\text { Hepatocellular Carcinoma after Ortho- } \\
\text { topic Liver Transplantation }\end{array}$ & 6 28-day cycles \\
\hline $\begin{array}{l}\text { Borthakur et al. } \\
(2011)-1\end{array}$ & Refractory or relapsed acute leukemias & $\begin{array}{l}\text { five days per week, every } \\
\text { week for a } 21 \text {-day cycle }\end{array}$ \\
\hline $\begin{array}{l}\text { Borthakur et al. } \\
(2011)-2\end{array}$ & Refractory or relapsed acute leukemias & 14 days every 21 days \\
\hline Crump et al. (2010)-1 & $\begin{array}{l}\text { Myelodysplastic syndrome or acute } \\
\text { myeloid leukemia }\end{array}$ & 28-day cycles \\
\hline Crump et al. (2010)-2 & $\begin{array}{l}\text { Myelodysplastic syndrome or acute } \\
\text { myeloid leukemia }\end{array}$ & 14-day rest every 28 days \\
\hline Furuse et al. (2008) & Hepatocellular carcinoma & $\begin{array}{l}\text { a single dose followed by a } \\
\text { 7-day wash-out period, and } \\
\text { then administrated twice } \\
\text { daily (bid) for a } 28 \text {-day cy- } \\
\text { cle }\end{array}$ \\
\hline
\end{tabular}


2. Trials details: irinotecan and S-1 example. 
TABle 2

Details on conditions, schedules and cycle definition for the 10 studies on the combination of irinotecan and S-1. S-1 was administered at a fixed dose of $80 \mathrm{mg} / \mathrm{m}^{2}$ per day in each study.

\begin{tabular}{|c|c|c|c|c|}
\hline Study & Conditions & S-1 schedule & $\begin{array}{l}\text { Irinotecan } \\
\text { schedule }\end{array}$ & Cycle \\
\hline Ogata et al. (2009) & $\begin{array}{ll}\begin{array}{l}\text { Advanced } \\
\text { cancer }\end{array} & \text { colorectal } \\
\end{array}$ & $\begin{array}{l}\text { From day } 3 \text { to } 7,10 \\
\text { to } 14 \text { and } 17 \text { to } 21\end{array}$ & Days $1,8,15$ & 28-day \\
\hline $\begin{array}{l}\text { Inokuchi et al. } \\
(2006)\end{array}$ & $\begin{array}{l}\text { Metastatic advanced } \\
\text { gastric cancer }\end{array}$ & From day 1 to 14 & Days 1 and 8 & 28-day \\
\hline Goya et al. (2012) & $\begin{array}{l}\text { Advanced non-small cell } \\
\text { lung cancer }\end{array}$ & From day 1 to 14 & Days 1 and 15 & 28-day \\
\hline $\begin{array}{l}\text { Takiuchi et al. } \\
(2005)\end{array}$ & $\begin{array}{l}\text { Advanced Gastric Can- } \\
\text { cer }\end{array}$ & From day 1 to 21 & Days 1 and 15 & 35-day \\
\hline $\begin{array}{l}\text { Ishimoto et al. } \\
(2009)\end{array}$ & $\begin{array}{l}\text { Advanced non-small cell } \\
\text { lung cancer }\end{array}$ & From day 1 to 14 & $\begin{array}{l}\text { Days } 1,8 \text {, and } \\
15\end{array}$ & 28-day \\
\hline $\begin{array}{l}\text { Kusaba et al. } \\
(2010)\end{array}$ & $\begin{array}{l}\text { Advanced } \\
\text { cancer }\end{array}$ & From day 1 to 14 & Days 1 and 8 & 21-day \\
\hline $\begin{array}{l}\text { Nakafusa et al. } \\
(2008)\end{array}$ & $\begin{array}{l}\text { Metastatic } \\
\text { cancer }\end{array}$ & From day 1 to 21 & Days 1 and 15 & 28-day \\
\hline $\begin{array}{l}\text { Shiozawa et al. } \\
(2009)\end{array}$ & $\begin{array}{l}\text { Advanced colorectal } \\
\text { cancer }\end{array}$ & From day 1 to 14 & Days 1 and 15 & 28-day \\
\hline Yoda et al. (2011) & $\begin{array}{l}\text { Previously treated ad- } \\
\text { vanced non-small cell } \\
\text { lung cancer }\end{array}$ & From day 1 to 14 & Days 1 and 8 & 21-day \\
\hline $\begin{array}{l}\text { Komatsu et al. } \\
(2010)\end{array}$ & $\begin{array}{l}\text { Advanced Gastric Can- } \\
\text { cer }\end{array}$ & From day 1 to 14 & Days 1 and 15 & 28-day \\
\hline
\end{tabular}


3. Sensitivity analysis tables. Tables 3 and 4 show the full results, in terms of percentage of MTD selection, of the sensitivity analysis performed. 
TABLE 3

Sensitivity analysis results: proportion of correct selection using 10 studies for each meta-analysis.

\begin{tabular}{|c|c|c|c|c|c|c|c|}
\hline & \multicolumn{7}{|c|}{ Dose levels } \\
\hline & 1 & 2 & 3 & 4 & 5 & 6 & 7 \\
\hline \multicolumn{8}{|l|}{ Scenario 1} \\
\hline MADF1 & 0.000 & 0.118 & 0.804 & 0.078 & 0.000 & 0.000 & 0.000 \\
\hline MADF2 & 0.000 & 0.071 & 0.690 & 0.232 & 0.007 & 0.000 & 0.000 \\
\hline MADF3 & 0.000 & 0.049 & 0.508 & 0.422 & 0.021 & 0.000 & 0.000 \\
\hline MADF4 & 0.000 & 0.045 & 0.456 & 0.406 & 0.088 & 0.004 & 0.001 \\
\hline \multicolumn{8}{|l|}{ Scenario 2} \\
\hline MADF1 & 0.000 & 0.000 & 0.079 & 0.908 & 0.013 & 0.000 & 0.000 \\
\hline MADF2 & 0.000 & 0.000 & 0.043 & 0.897 & 0.060 & 0.000 & 0.000 \\
\hline MADF3 & 0.000 & 0.000 & 0.017 & 0.882 & 0.101 & 0.000 & 0.000 \\
\hline MADF4 & 0.000 & 0.000 & 0.026 & 0.840 & 0.131 & 0.003 & 0.000 \\
\hline \multicolumn{8}{|l|}{ Scenario 3} \\
\hline MADF1 & 0.000 & 0.000 & 0.000 & 0.229 & 0.735 & 0.036 & 0.000 \\
\hline MADF2 & 0.000 & 0.000 & 0.000 & 0.101 & 0.709 & 0.185 & 0.005 \\
\hline MADF3 & 0.000 & 0.000 & 0.000 & 0.056 & 0.773 & 0.171 & 0.000 \\
\hline MADF4 & 0.000 & 0.000 & 0.000 & 0.061 & 0.554 & 0.329 & 0.056 \\
\hline \multicolumn{8}{|l|}{ Scenario 4} \\
\hline MADF1 & 0.000 & 0.000 & 0.000 & 0.002 & 0.354 & 0.638 & 0.006 \\
\hline MADF2 & 0.000 & 0.000 & 0.000 & 0.000 & 0.132 & 0.758 & 0.110 \\
\hline MADF3 & 0.000 & 0.000 & 0.000 & 0.000 & 0.139 & 0.819 & 0.042 \\
\hline MADF4 & 0.000 & 0.000 & 0.000 & 0.000 & 0.078 & 0.585 & 0.337 \\
\hline \multicolumn{8}{|l|}{ Scenario 5} \\
\hline MADF1 & 0.000 & 0.001 & 0.216 & 0.747 & 0.036 & 0.000 & 0.000 \\
\hline MADF2 & 0.000 & 0.001 & 0.105 & 0.734 & 0.156 & 0.004 & 0.000 \\
\hline MADF3 & 0.000 & 0.000 & 0.043 & 0.653 & 0.303 & 0.001 & 0.000 \\
\hline MADF4 & 0.000 & 0.000 & 0.051 & 0.575 & 0.337 & 0.037 & 0.000 \\
\hline \multicolumn{8}{|l|}{ Scenario 6} \\
\hline MADF1 & 0.000 & 0.000 & 0.053 & 0.918 & 0.029 & 0.000 & 0.000 \\
\hline MADF2 & 0.000 & 0.000 & 0.028 & 0.892 & 0.080 & 0.000 & 0.000 \\
\hline MADF3 & 0.000 & 0.000 & 0.009 & 0.820 & 0.171 & 0.000 & 0.000 \\
\hline MADF4 & 0.000 & 0.000 & 0.022 & 0.798 & 0.172 & 0.008 & 0.000 \\
\hline \multicolumn{8}{|l|}{ Scenario 7} \\
\hline MADF1 & 0.000 & 0.000 & 0.000 & 0.208 & 0.751 & 0.041 & 0.000 \\
\hline MADF2 & 0.000 & 0.000 & 0.001 & 0.088 & 0.696 & 0.210 & 0.005 \\
\hline MADF3 & 0.000 & 0.000 & 0.000 & 0.049 & 0.753 & 0.198 & 0.000 \\
\hline MADF4 & 0.000 & 0.000 & 0.000 & 0.050 & 0.534 & 0.349 & 0.067 \\
\hline \multicolumn{8}{|l|}{ Scenario 8} \\
\hline MADF1 & 0.000 & 0.000 & 0.003 & 0.338 & 0.630 & 0.029 & 0.000 \\
\hline MADF2 & 0.000 & 0.000 & 0.002 & 0.149 & 0.647 & 0.192 & 0.010 \\
\hline MADF3 & 0.000 & 0.000 & 0.000 & 0.085 & 0.730 & 0.185 & 0.000 \\
\hline MADF4 & 0.000 & 0.000 & 0.001 & 0.077 & 0.511 & 0.339 & 0.072 \\
\hline \multicolumn{8}{|l|}{ Scenario 9} \\
\hline MADF1 & 0.000 & 0.000 & 0.001 & 0.167 & 0.773 & 0.059 & 0.000 \\
\hline MADF2 & 0.000 & 0.000 & 0.000 & 0.091 & 0.699 & 0.205 & 0.005 \\
\hline MADF3 & 0.000 & 0.000 & 0.000 & 0.044 & 0.771 & 0.185 & 0.000 \\
\hline MADF4 & 0.000 & 0.000 & 0.000 & 0.048 & 0.578 & 0.331 & 0.043 \\
\hline
\end{tabular}


TABle 4

Sensitivity analysis results: proportion of correct selection using 5 studies for each meta-analysis.

\begin{tabular}{|c|c|c|c|c|c|c|c|}
\hline & \multicolumn{7}{|c|}{ Dose levels } \\
\hline & 1 & 2 & 3 & 4 & 5 & 6 & 7 \\
\hline \multicolumn{8}{|l|}{ Scenario 1} \\
\hline MADF1 & 0.004 & 0.213 & 0.637 & 0.138 & 0.008 & 0.000 & 0.000 \\
\hline MADF2 & 0.005 & 0.142 & 0.585 & 0.235 & 0.030 & 0.003 & 0.000 \\
\hline MADF3 & 0.007 & 0.117 & 0.463 & 0.361 & 0.051 & 0.001 & 0.000 \\
\hline MADF4 & 0.003 & 0.112 & 0.457 & 0.317 & 0.093 & 0.017 & 0.001 \\
\hline \multicolumn{8}{|l|}{ Scenario 2} \\
\hline MADF1 & 0.000 & 0.002 & 0.163 & 0.796 & 0.038 & 0.001 & 0.000 \\
\hline MADF2 & 0.000 & 0.002 & 0.095 & 0.817 & 0.084 & 0.002 & 0.000 \\
\hline MADF3 & 0.000 & 0.000 & 0.046 & 0.801 & 0.152 & 0.001 & 0.000 \\
\hline MADF4 & 0.000 & 0.000 & 0.058 & 0.766 & 0.170 & 0.005 & 0.001 \\
\hline \multicolumn{8}{|l|}{ Scenario 3} \\
\hline MADF1 & 0.000 & 0.000 & 0.011 & 0.333 & 0.588 & 0.068 & 0.000 \\
\hline MADF2 & 0.000 & 0.000 & 0.003 & 0.206 & 0.599 & 0.178 & 0.014 \\
\hline MADF3 & 0.000 & 0.000 & 0.001 & 0.132 & 0.659 & 0.205 & 0.003 \\
\hline MADF4 & 0.000 & 0.000 & 0.001 & 0.116 & 0.555 & 0.253 & 0.075 \\
\hline \multicolumn{8}{|l|}{ Scenario 4} \\
\hline MADF1 & 0.000 & 0.000 & 0.001 & 0.034 & 0.456 & 0.488 & 0.021 \\
\hline MADF2 & 0.000 & 0.000 & 0.000 & 0.010 & 0.250 & 0.634 & 0.106 \\
\hline MADF3 & 0.000 & 0.000 & 0.000 & 0.001 & 0.246 & 0.673 & 0.080 \\
\hline MADF4 & 0.000 & 0.000 & 0.000 & 0.002 & 0.151 & 0.568 & 0.279 \\
\hline \multicolumn{8}{|l|}{ Scenario 5} \\
\hline MADF1 & 0.000 & 0.019 & 0.284 & 0.614 & 0.079 & 0.004 & 0.000 \\
\hline MADF2 & 0.000 & 0.015 & 0.187 & 0.589 & 0.191 & 0.016 & 0.002 \\
\hline MADF3 & 0.000 & 0.011 & 0.097 & 0.578 & 0.301 & 0.013 & 0.000 \\
\hline MADF4 & 0.000 & 0.009 & 0.104 & 0.531 & 0.286 & 0.065 & 0.005 \\
\hline \multicolumn{8}{|l|}{ Scenario 6} \\
\hline MADF1 & 0.000 & 0.000 & 0.129 & 0.812 & 0.057 & 0.002 & 0.000 \\
\hline MADF2 & 0.000 & 0.000 & 0.082 & 0.802 & 0.111 & 0.005 & 0.000 \\
\hline MADF3 & 0.000 & 0.000 & 0.045 & 0.769 & 0.179 & 0.007 & 0.000 \\
\hline MADF4 & 0.000 & 0.000 & 0.053 & 0.731 & 0.186 & 0.028 & 0.002 \\
\hline \multicolumn{8}{|l|}{ Scenario 7} \\
\hline MADF1 & 0.000 & 0.000 & 0.013 & 0.317 & 0.600 & 0.070 & 0.000 \\
\hline MADF2 & 0.000 & 0.000 & 0.004 & 0.170 & 0.610 & 0.202 & 0.014 \\
\hline MADF3 & 0.000 & 0.000 & 0.001 & 0.123 & 0.658 & 0.214 & 0.004 \\
\hline MADF4 & 0.000 & 0.000 & 0.002 & 0.104 & 0.516 & 0.290 & 0.088 \\
\hline \multicolumn{8}{|l|}{ Scenario 8} \\
\hline MADF1 & 0.000 & 0.000 & 0.042 & 0.413 & 0.500 & 0.044 & 0.001 \\
\hline MADF2 & 0.000 & 0.000 & 0.015 & 0.232 & 0.554 & 0.181 & 0.018 \\
\hline MADF3 & 0.000 & 0.001 & 0.005 & 0.150 & 0.645 & 0.192 & 0.007 \\
\hline MADF4 & 0.000 & 0.000 & 0.005 & 0.141 & 0.514 & 0.248 & 0.092 \\
\hline \multicolumn{8}{|l|}{ Scenario 9} \\
\hline MADF1 & 0.000 & 0.000 & 0.008 & 0.271 & 0.635 & 0.085 & 0.001 \\
\hline MADF2 & 0.000 & 0.000 & 0.005 & 0.173 & 0.622 & 0.189 & 0.011 \\
\hline MADF3 & 0.000 & 0.000 & 0.002 & 0.114 & 0.684 & 0.196 & 0.004 \\
\hline MADF4 & 0.000 & 0.000 & 0.005 & 0.095 & 0.552 & 0.279 & 0.069 \\
\hline
\end{tabular}

imsart-aoas ver. 2014/10/16 file: supp_mat_rev2.tex date: July 28, 2020 
TABLE 5

Settings and parameters in scenario 10, 11 and 12.

\begin{tabular}{cccc}
\hline Scenario & $\begin{array}{c}\text { Fixed effect } \\
\text { true } \mathbf{p}^{\star}\end{array}$ & Random effect & Studies design \\
\hline 10 & a) & $\Sigma=\left[\exp \left(-\frac{\delta_{i, j}^{2}}{2 l^{2}}\right) \sigma_{m}^{2}\right], \sigma_{m}=0.3$ & CRM and $3+3$ \\
11 & a) & $\Sigma=\left[\exp \left(-\frac{\delta_{i, j}^{2}}{2 l^{2}}\right) \sigma_{m}^{2}\right], \sigma_{m}=0.3$ & only 3+3 design \\
12 & a) & $\Sigma=\left[\exp \left(-\frac{\delta_{i, j}^{2}}{2 l^{2}}\right) \sigma_{m}^{2}\right], \sigma_{m}=0.3$ & only CRM design \\
\hline
\end{tabular}

4. New scenarios. In this section, 3 further scenarios are investigated. They are variations of Scenario 1, with which share the fixed effect probability set (a), that is, $\mathbf{p}^{\star}=(0.15,0.20,0.33,0,45,0.55,0.60,0.65)$. Details are given in Table 5 and results in Table 6 . 
TABLE 6

Proportion of dose selection using 10 studies in each meta-analysis. The proportion of correct MTD selection (PCS) in each scenario is written in bold. \#patients: median (first quartile - third quartile) number of patients allocated to each dose.

\begin{tabular}{|c|c|c|c|c|c|c|c|}
\hline & \multicolumn{7}{|c|}{ Dose levels } \\
\hline & 1 & 2 & 3 & 4 & 5 & 6 & 7 \\
\hline \multicolumn{8}{|l|}{ Scenario 10} \\
\hline MADF & 0.000 & 0.080 & 0.574 & 0.340 & 0.006 & 0.000 & 0.000 \\
\hline MADF1 & 0.000 & 0.108 & 0.798 & 0.094 & 0.000 & 0.000 & 0.000 \\
\hline MADF2 & 0.000 & 0.064 & 0.660 & 0.266 & 0.010 & 0.000 & 0.000 \\
\hline MADF3 & 0.000 & 0.054 & 0.455 & 0.460 & 0.031 & 0.000 & 0.000 \\
\hline MADF4 & 0.000 & 0.040 & 0.422 & 0.410 & 0.123 & 0.003 & 0.002 \\
\hline $\mathrm{ZKO}$ & 0.024 & 0.191 & 0.478 & 0.265 & 0.037 & 0.003 & 0.002 \\
\hline \#patients & $31(23,41)$ & $31(23,40)$ & $53(43,63)$ & $16(10,24)$ & $6(3,12)$ & $2(0,6)$ & $0(0,3)$ \\
\hline \multicolumn{8}{|l|}{ Scenario 11} \\
\hline MADF & 0.000 & 0.047 & 0.555 & 0.391 & 0.007 & 0.000 & 0.000 \\
\hline MADF1 & 0.000 & 0.057 & 0.748 & 0.193 & 0.002 & 0.000 & 0.000 \\
\hline MADF2 & 0.000 & 0.047 & 0.638 & 0.301 & 0.013 & 0.001 & 0.000 \\
\hline MADF3 & 0.000 & 0.028 & 0.466 & 0.485 & 0.021 & 0.000 & 0.000 \\
\hline MADF4 & 0.000 & 0.015 & 0.504 & 0.416 & 0.061 & 0.004 & 0.000 \\
\hline $\mathrm{ZKO}$ & 0.015 & 0.266 & 0.496 & 0.191 & 0.030 & 0.002 & 0.000 \\
\hline \#patients & $40(30,52)$ & $45(34,58)$ & $48(37.75,63)$ & $32(22,45)$ & $24(15,35)$ & $0(0,4)$ & $0(0,2)$ \\
\hline \multicolumn{8}{|l|}{ Scenario 12} \\
\hline MADF & 0.001 & 0.089 & 0.567 & 0.338 & 0.005 & 0.000 & 0.000 \\
\hline MADF1 & 0.000 & 0.145 & 0.755 & 0.100 & 0.000 & 0.000 & 0.000 \\
\hline MADF2 & 0.000 & 0.078 & 0.626 & 0.272 & 0.024 & 0.000 & 0.000 \\
\hline MADF3 & 0.000 & 0.052 & 0.431 & 0.467 & 0.050 & 0.000 & 0.000 \\
\hline MADF4 & 0.000 & 0.046 & 0.412 & 0.406 & 0.122 & 0.012 & 0.002 \\
\hline $\mathrm{ZKO}$ & 0.011 & 0.146 & 0.490 & 0.293 & 0.058 & 0.002 & 0.000 \\
\hline \#patients & $27(21,33)$ & $21(18,27)$ & $21(15,24)$ & $12(9,15)$ & $9(6,15)$ & $0(0,3)$ & $0(0,0)$ \\
\hline
\end{tabular}


TABLE 7

Proportion of dose selection using 5 studies in each meta-analysis. The proportion of correct MTD selection (PCS) in each scenario is written in bold. \#patients: median (first quartile - third quartile) number of patients allocated to each dose.

\begin{tabular}{|c|c|c|c|c|c|c|c|}
\hline & \multicolumn{7}{|c|}{ Dose levels } \\
\hline & 1 & 2 & 3 & 4 & 5 & 6 & 7 \\
\hline \multicolumn{8}{|l|}{ Scenario 10} \\
\hline MADF & 0.005 & 0.160 & 0.480 & 0.324 & 0.031 & 0.000 & 0.000 \\
\hline MADF1 & 0.002 & 0.211 & 0.625 & 0.157 & 0.005 & 0.000 & 0.000 \\
\hline MADF2 & 0.002 & 0.146 & 0.557 & 0.253 & 0.037 & 0.005 & 0.000 \\
\hline MADF3 & 0.004 & 0.111 & 0.453 & 0.369 & 0.062 & 0.001 & 0.000 \\
\hline MADF4 & 0.002 & 0.106 & 0.436 & 0.335 & 0.101 & 0.019 & 0.001 \\
\hline ZKO & 0.042 & 0.166 & 0.365 & 0.302 & 0.077 & 0.035 & 0.013 \\
\hline \#patients & $15(9,23)$ & $16(10,23)$ & $28(21,36)$ & $8(3,14)$ & $3(0,6)$ & $0(0,3)$ & $0(0,0)$ \\
\hline \multicolumn{8}{|l|}{ Scenario 11} \\
\hline MADF & 0.003 & 0.125 & 0.439 & 0.404 & 0.029 & 0.000 & 0.000 \\
\hline MADF1 & 0.002 & 0.130 & 0.615 & 0.247 & 0.006 & 0.000 & 0.000 \\
\hline MADF2 & 0.003 & 0.130 & 0.531 & 0.306 & 0.029 & 0.001 & 0.000 \\
\hline MADF3 & 0.002 & 0.106 & 0.397 & 0.423 & 0.071 & 0.001 & 0.000 \\
\hline MADF4 & 0.000 & 0.081 & 0.425 & 0.365 & 0.120 & 0.009 & 0.000 \\
\hline ZKO & 0.044 & 0.167 & 0.352 & 0.333 & 0.097 & 0.006 & 0.001 \\
\hline \#patients & $19(11,29)$ & $21(13,32)$ & $24(15,34)$ & $15(8,24)$ & $12(4,20)$ & $0(0,2)$ & $0(0,0)$ \\
\hline \multicolumn{8}{|l|}{ Scenario 12} \\
\hline MADF & 0.025 & 0.168 & 0.445 & 0.315 & 0.047 & 0.000 & 0.000 \\
\hline MADF1 & 0.013 & 0.260 & 0.559 & 0.160 & 0.008 & 0.000 & 0.000 \\
\hline MADF2 & 0.014 & 0.175 & 0.488 & 0.264 & 0.057 & 0.002 & 0.000 \\
\hline MADF3 & 0.019 & 0.134 & 0.382 & 0.365 & 0.099 & 0.001 & 0.000 \\
\hline MADF4 & 0.010 & 0.143 & 0.377 & 0.309 & 0.141 & 0.015 & 0.005 \\
\hline ZKO & 0.052 & 0.147 & 0.328 & 0.328 & 0.131 & 0.012 & 0.002 \\
\hline \#patients & $12(9,15)$ & $12(9,15)$ & $9(6,15)$ & $6(3,9)$ & $3(3,9)$ & $0(0,0)$ & $0(0,0)$ \\
\hline
\end{tabular}

